\title{
Phytotherapeutische Bittermittel
}

\author{
Reinhard Saller, Jörg Melzer, Bernhard Uehleke, Matthias Rostock \\ Institut für Naturheilkunde, Department für Innere Medizin, UniversitätsSpital, Zürich, Schweiz
}

A ls phytotherapeutische Bittermittel werden in der Regel Arzneidrogen bzw. deren einfache galenische Zubereitungen (z.B. Drogenpulver, Tees, Tinkturen, Extrakte, Pflanzensäfte) verwendet. Für Wirksamkeit und Dosierung wird zumeist der bereits in der Mundhöhle wahrnehmbare Bittergeschmack solcher einfachen Zubereitungen therapeutisch mit ausgenutzt. Diese phytotherapeutischen Wirkstoffe (Zubereitungen) enthalten Komponenten mit Bitterstoffcharakter (Bitterstoffe). Bitterstoffe sind verschiedene Substanzgruppen mit ganz differenten Grundstrukturen, die alle das Geschmacksmerkmal „bitter“ (amarum) aufweisen. Zahlreiche Bitterstoffe besitzen eine niedrige Entdeckungsschwelle. $\mathrm{Zu}$ den Bitterstoffen gehören u.a. Monoterpene, Sesquiterpene, Diterpene, Triterpene, Alkaloide, Iridoidglykoside, Secoiridoidglykoside, Phloroglucinderivate, Peptide, Aminosäuren. Entsprechend dieser Vielfalt von Bitterstoffen besitzt ein grosses Spektrum von Arzneidrogen einen wenngleich sehr unterschiedlichen Bitterstoffcharakter. Dieser wird mit Hilfe des Bitterwertes zumeist noch sensorisch abgeschätzt. Eine Auswahl von Drogen mit Bitterwerten ist in Tab. 1 zusammengestellt.

An der Wahrnehmung von „bitter“ sind wahrscheinlich mehr als 50 verschiedene Rezeptoren und mehr als 20 Gene beteiligt. Es besteht eine grosse Gen- und Rezeptorenvielfalt [6,16]. Die zahlreichen Rezeptortypen ermöglichen eine Vielzahl chemisch unterschiedlicher Bitterstoffe zu erkennen. Dadurch ergibt sich ein relativ grosses Wirkungsspektrum. Insgesamt sind phytotherapeutische Bitterdrogen komplex zusammengesetzte Vielstoffgemische. Dementsprechend können die einzelnen Bittermittel neben ihrer Bitterkeit

Phytotherapeutische Bitterdrogen sind komplex zusammengesetzte Vielstoffgemische. Bei den enthaltenden Bitterstoffen handelt es sich um Substanzgruppen mit verschiedenen Grundstrukturen, die alle durch das Geschmacksmerkmal "bitter" (amarum) charakterisiert sind und sensorisch mittels des Bitterwertes eingeschätzt werden. Dementsprechend können Bittermittel neben ihrer Bitterkeit ein insgesamt vielfältiges Wirkungsspektrum aufweisen. Therapeutisch mit ausgenutzt wird der bereits in der Mundhöhle wahrnehmbare Bittergeschmack. Derzeit ist die gängige und zum Teil monografierte therapeutische Anwendung von Bittermitteln mit ihren appetitanregenden, sekretionsfördernden und motilitätssteigernden Wirkungen eingeengt auf die Behandlung von Appetitlosigkeit, dyspeptischen Beschwerden bzw. Magen-Darm-Beschwerden und teilweise Störungen des Galleflusses. Ergänzend dazu besitzen Bittermittel ein antidepressives und konstitutionstherapeutisches Potential und können, bedingt durch die Einflüsse des Magen-Darm-Traktes auf das Gesamtbefinden, mit Erfolg auch bei Müdigkeit, Erschöpfung, Stress oder allgemein bei somatoformen Störungen eingesetzt werden.

Schlüselwörter: Phytotherapie, Bittermittel, Tonikum, Adaptogen, Amarum, Vielstoffgemisch, Magen-Darm-Beschwerden, somatoforme Störungen, Konstitutionstherapie.

\section{Bitter Herbal Drugs}

Bitter herbal drugs are complex multicompounds containing bitter ingredients which are groups of substances with various basic structures, all characterized by their bitter (amarum) taste and sensorily classified according to their bitterness value. Bitters share their bitter taste but may have manifold activities. Their bitter taste which is sensed first in the oral cavity is also used for therapeutic purposes. Currently, the therapeutic use and therapeutic application partly based on monographs of bitters with their appetizing, secretion-inducing and motility-increasing effects are restricted to the treatment of dyspeptic or gastrointestinal complaints and, in part, to the treatment of bile flow disturbances. Additionally, bitters have an antidepressive and constitutional therapeutic potential and can - due to the influences of the gastrointestinal tract on the overall state of health - be successfully used against fatigue, exhaustion, stress or generally in somatoform disorders.

Key words: Phytotherapy, bitter herbal drugs, tonic, adaptogenic, multicompound, gastrointestinal complaints, somatoform disorder, constitutional therapy

ein differenziertes und insgesamt vielfältiges Wirkungsspektrum aufweisen $[7,9,28,29,33]$.

Derzeit ist die gängige und z.T. monografierte therapeutische Anwendung von Bittermitteln mit ihren appetitanregenden, sekretionsfördernden und motilitätssteigernden Wirkungen eingeengt auf die Behandlung von Appetitlosigkeit, dyspeptischen Beschwerden bzw. Magen-Darm-Beschwerden und teilweise Störungen des Galleflus- ses. Damit wird aber das Potential von phytotherapeutischen Bittermitteln keinesfalls ausgeschöpft [27]. Die Wahrnehmung „bitter“ ist entwicklungsgeschichtlich mit „Gefahr” verbunden. Dies weist auf auf die Bedeutung von „bitter“ und eine Reihe durch Bitterwahrnehmung ausgelöste physiologische Reaktionen hin. Das zumeist unterschätzte therapeutische Potential von Bitterstoffdrogen lässt sich z.B. in einer kontrollierten Studie erkennen, 
Tab. 1. Bitterstoffdrogen (Auswahl): Abschätzung der Bitterwerte

\begin{tabular}{ll}
\hline Bitterholz & $40000-50000$ \\
\hline Enzianwurzel & $10000-25000$ \\
\hline Wermutkraut & $10000-25000$ \\
\hline Artischockenblätter & $5000-15000$ \\
\hline Teufelskrallenwurzel & $5000-15000$ \\
\hline Fieberkleeblätter & $4000-10000$ \\
\hline Tausendgüldenkraut & $2000-10000$ \\
\hline Andornkraut & 3000 \\
\hline Pomeranzenschalen & $1000-2500$ \\
\hline Benediktenkraut & $1000-2500$ \\
\hline Chinarinde & 1000 \\
\hline Löwenzahnwurzel & $>100$ \\
\hline
\end{tabular}

Die Bitterkeit wird sensorisch abgeschätzt, z.B. durch Bestimmung des Bitterwertes. Dieser ist der reziproke Wert der Konzentration eines Bitterstoffes bzw. einer Bitterdroge in einem Extrakt (ausgehend von $1 \mathrm{~g}$ ), der gerade noch als bitter schmeckend empfunden wird.

Tab. 2. Einteilung von Amara entsprechend weiteren Komponenten des phytotherapeutischen Wirkstoffes (zusätzliche Wirkungen, erweitertes Wirkungsspektrum)

Amara pura [Amara simplicia, Amara tonica] (nur bzw. vorwiegend Bitterstoffe) Beispiele: Enzianwurzel, Bitterholz, Bitterklee, Fieberklee, Tausendgüldenkraut

\section{Amara aromatica (Bitterstoffe und ätherisches Öl)}

Beispiele: Angelikawurzel, Condurangorinde, Hopfenzapfen, Kalmuswurzelstock, Kardobenediktenkraut, Orangenschalen, Pomeranzenschalen, Wermutkraut

Amara adstringentia (Bitterstoffe und Gerbstoffe, Tannine)

Beispiele: Chinarinde, Condurangorinde

Amara mucilagenosa (Bitterstoffe und bedeutsame Mengen Schleimstoffe)

Beispiele: Lichen islandicus

Amara acria (Bitterstoffe und bedeutsame Mengen Scharfstoffe)

Beispiele: Ingwerrhizom, Galgantwurzelstock

Bitterkomponenten sind in einer Reihe weiterer Drogen enthalten. Dazu gehören u.a. Beifusskraut, Birkenblätter, bittere Schleifenblume, Mariendistelfrüchte, Mariendistelkraut, Primelblüten, Primelwurzel, Rosmarinblätter, Salbeiblätter, Schafgarbenblüten, Schafgarbenkraut. Auch bei ihrer Verwendung lässt sich der Bitterstoffcharakter zusätzlich zu anderen Wirkungen therapeutisch nutzen.

Tab. 3. Wirkstoffbezogene empirische Charakterisierung ausgewählter Tinkturen (Bittermittel, Reihung nach Bitterkeit und "Wärme")

Tinctura Absinthii (Wermutkrauttinktur)

Aromatisches Bittermittel, Stomachikum, Gallemittel, Magentonikum, allgemeines Tonikum

Tinctura Gentianae (Enziantinktur)

Amarum, Stomachikum, Gallemittel, Magentonikum, allgemeines Tonikum

Tinctura Cynarae (Artischockentinktur)

Amarum, Lebermittel, Gallemittel

Tinctura Taraxaci - Löwenzahntinktur

Amarum, Magentonikum, Gallemittel, Aquaretikum, Stoffwechselmittel

Tinctura millefolii (Schafgarbentinktur)

Karminativum, Spasmolytikum, Gallemittel, Stoffwechselmittel, Frauenmittel in der Wermutpulver (1.5 g/Tag) eine ausgeprägte antientzündliche, steroidmindernde, generell symptomlindernde (u.a. auch dezidiert antidepressive) Wirksamkeit bei Patienten mit einem M. Crohn und einer eingreifenden immunsuppressiven Behandlung zeigte [14].

\section{Einteilung von Bitterstoffdrogen}

Die einzelnen phytotherapeutischen Bittermittel werden nach nach den zusätzlichen Komponenten ihrer Wirkstoffe mit Vielstoffcharakter (neben den Bitterkomponenten) bzw. sensorischen Qualitäten in verschiedene Gruppen von Amara eingereiht (siehe Tab. 2) $[19,20]$. Gerade auch die komplex zusammengesetzten Amara besitzen neben den Bitterstoffen noch zusätzliche Wirkungen, die in der Behandlung unterschiedlicher Störungen nützlich sein können (z.B. spasmolytische und antibakterielle Effekte von ätherischen Ölen in Amara aromatica bei dyspeptischen Störungen) [20]). Eine Auswahl von Bitterstofftinkturen mit einer therapeutisch-empirischen Charakterisierung entsprechend der möglichen Anwendungsgebiete ist in Tabelle 3 zusammengefasst.

Amara-Aromatika stellen z.B. einen wesentlichen Teil der Stomachika (,Magenmittel“, appetit- und verdauungsanregende Mittel) dar [5,7,8,19, 20]. „Magen“ ist in diesem Zusammenhang nicht ausschliesslich anatomisch, sondern in einem erweiterten Sinn, gebraucht. Mit solchen Stomachika lassen sich in diesem Kontext auch eine Reihe assoziierter extraintestinaler Beschwerden behandeln. Ebenso lassen sich Müdigkeit und Abgeschlagenheit mit energetisierenden Bittermitteln behandeln.

\section{Bitterstoffdrogen und depressive Verstimmungen}

Über lange Zeiträume wurden depressive Verstimmungen mit individuell ausgewählten Bitterstoffdrogen behandelt. Zur Möglichkeit antidepressiver Wirkungen von Bitterdrogen liegen 
Tab. 4. Einteilung von bitterstoffhaltigen Zubereitungen entsprechend einer empirisch orientierten Beurteilung von Qualitäten, z.B. Tinkturen

\begin{tabular}{|c|c|}
\hline Wärme: & „heisse” - „neutrale” - „kalte“ Tinkturen \\
\hline $\begin{array}{l}\text { Tonus (allgemein, Magen- } \\
\text { Darm-Trakt): }\end{array}$ & $\begin{array}{l}\text { hypertonisch - dystonisch - normotonisch - } \\
\text { hypotonisch - atonisch }\end{array}$ \\
\hline Energie: & unterschiedlich stark „energetisierende“ Tinkturen \\
\hline Wahrnehmung: & $\begin{array}{l}\text { teilweise subjektiv und individuell, alters- und } \\
\text { teilweise geschlechtsabhängig }\end{array}$ \\
\hline \multicolumn{2}{|c|}{$\begin{array}{l}\text { Unterschiedliches krankheitsbezogenes Wirkungsspektrum der einzelnen Tinkturen } \\
\text { Therapeutische Konsequenzen: individuelle Dosierungen und Dosisanpassungen }\end{array}$} \\
\hline
\end{tabular}

Tab. 5. Konstitutionstypologische Reihung von Bittertinkturen (z.B. Urtinkturen) als „Energiemittel"

bei hitzigem, quirligem, nervösem Typus/Zustand:
$\begin{aligned} \text { Tinctura Taraxaci } & \text { (Löwenzahn, Drogen: Wurzel, Kraut) }\end{aligned}$

bei eher ausgeglichenem Typus/Zustand: Tinctura Cynarae (Artischocke, Droge: Blätter)

bei kaltem („,energiearmem“), eher behäbigem, wässrigem Typus/Zustand: Tinctura Gentianae (gelber Enzian, Droge: Wurzel)

bei kaltem („energiearmem“), behäbigem, wässrigem Typus/Zustand: Tinctura Absinthii (Wermut, Droge: Kraut)

Als einen Anhaltspunkt für die Einteilung weiterer Bittertinkturen in dieses konstitutionstherapeutische Schema lässt sich die jeweilige „Bitterkeit" heranziehen.

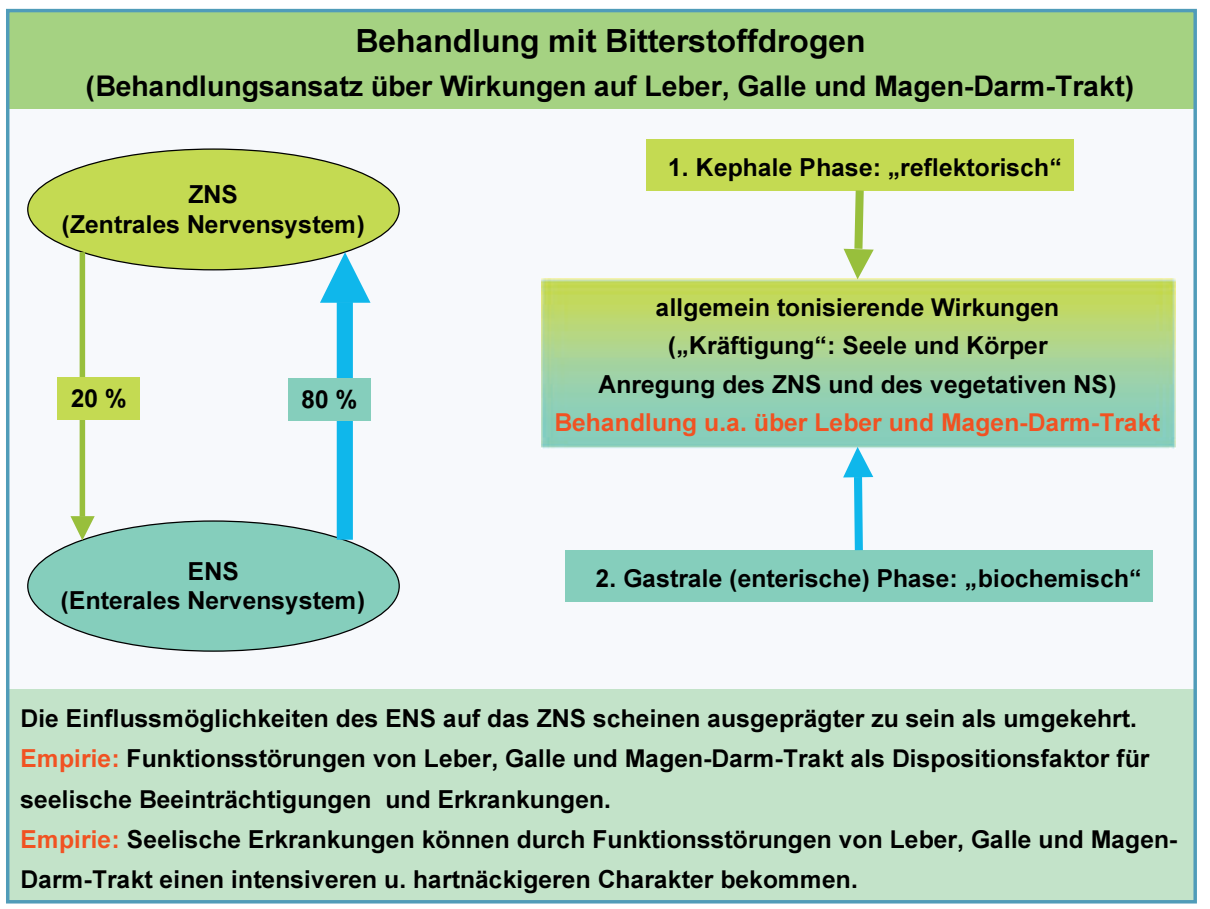

Abb. 1. Behandlung mit Bitterstoffdrogen (Behandlungsansatz über Wirkungen auf Leber, Galle und Magen-Darm-Trakt) mittlerweile auch erste moderne klinische Studien vor, die neben anderen symptomorientierten Wirkungen von Bittermitteln auf ein ausgeprägtes antidepressives Potential hinweisen. So zeigte eine kontrollierte klinische Studie bei PatientInnen mit einem aktiven M. Crohn, dass ein einfaches Drogenpräparat mit Wermutpulver (Wermutkraut) - neben klinisch relevanten antiinflammatorischen Wirkungen und einer ausgeprägten symptomatischen Besserung - bedeutsame antidepressive Wirkungen besitzen kann [14].

Therapeutische Empirie weist darauf hin, dass sich die verschiedenen phytotherapeutischen Bittermittel entsprechend ihrer Bitterkeit gruppieren lassen [19,20,21]. Dadurch wird es möglich, solche Bittermittel unter konstitutionstypologischen Gesichtspunkten individuell differenziert anzuwenden. „Bitterkeit“ steht dabei u.a. in metaphorischer Hinsicht für den jeweiligen „Energiegehalt“ der verschiedenen Zubereitungen (v.a. Tinkturen, aber auch Teezubereitungen und einfache Drogenzubereitungen wie z.B. Presstabletten). Tabelle 5 zeigt eine solche Reihung von Bittermitteln am Beispiel ausgewählter Bittertinkturen. Neben Wermutkraut und Artischockenblättern gehören zu solchen Bittermitteln u.a. Enzianwurzel (Gentiana lutea) $[13,15]$ und Löwenzahnwurzel bzw. Löwenzahnwurzel und -kraut (Taraxacum officinale) [10,22,25].

\section{Bitterstoffdrogen als Allgemeinbehandlung und Tonika}

Die Wahrnehmung von „bitter“ erfolgt nicht nur im Bereich der Mundhöhle. In den letzten Jahren wurden Bitterrezeptoren im gesamten Magen-DarmTrakt entdeckt $[17,24]$. Diese Entdeckungen können erklären, dass Bitterwirkungen nicht nur quasi reflektorisch über die Mundhöhle, sondern auch sozusagen biochemisch bei gastroenteraler Aufnahme, ausgelöst werden. Mittlerweile gilt es als gesichert, dass die Einflüsse vom Magen-DarmTrakt auf das Gehirn wesentlich ausgeprägter sein können als umgekehrt (Abb. 1). Damit bietet sich zumindest 

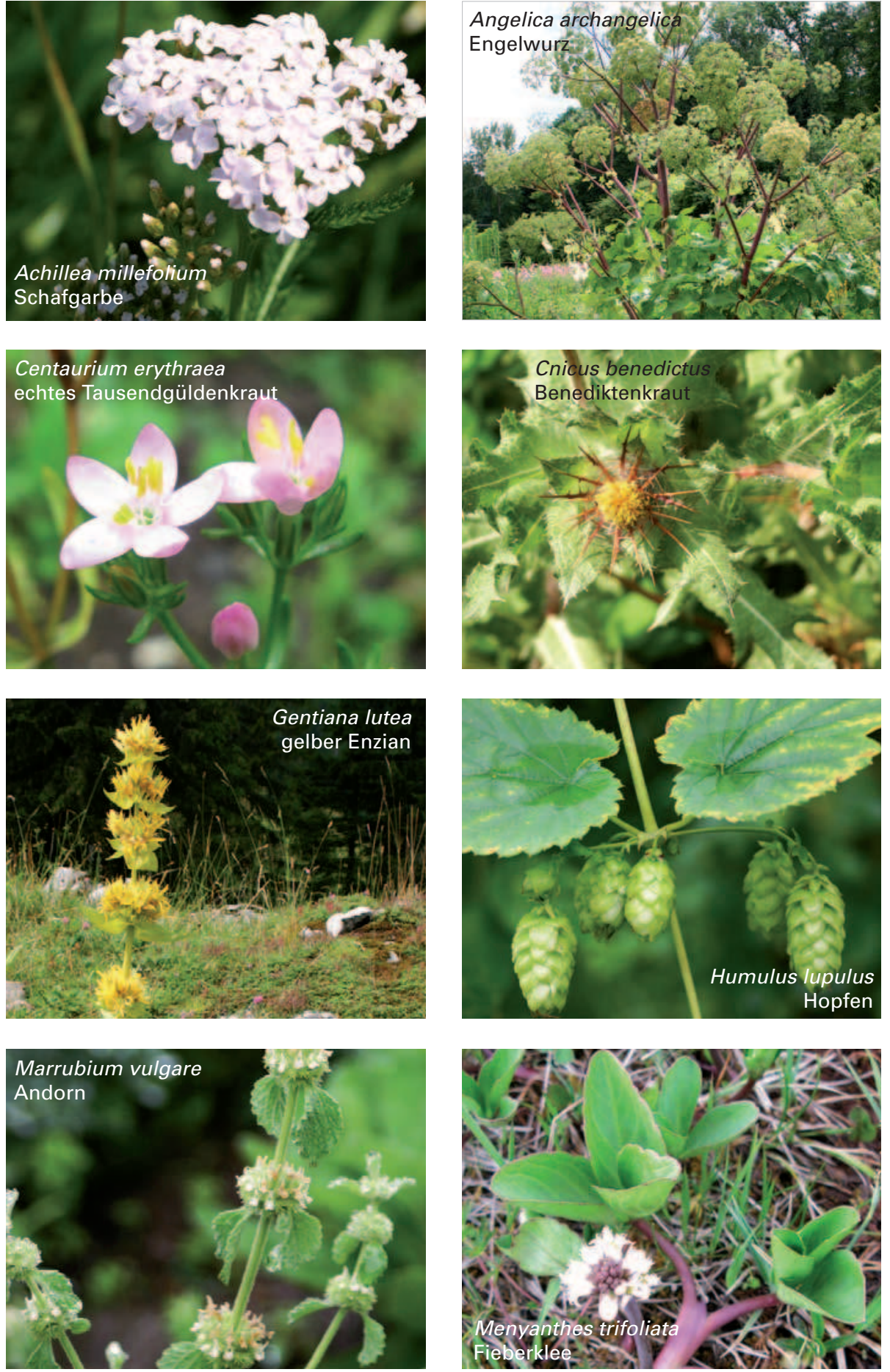
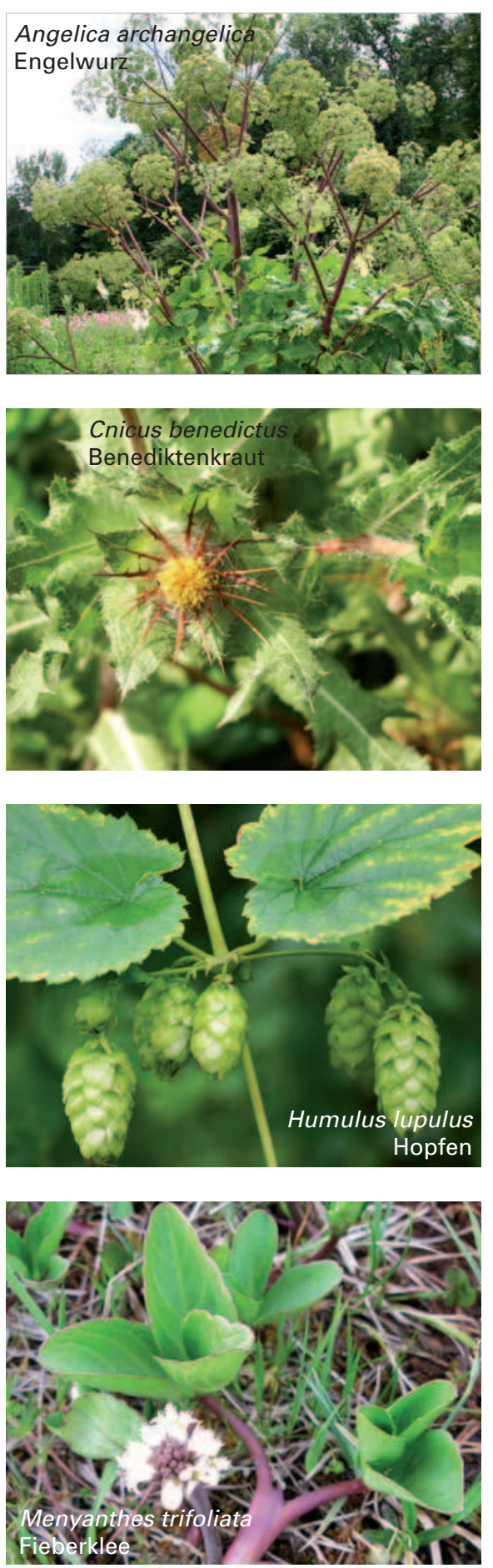

Auswahl an Pflanzen, aus denen Bitterstoffdrogen gewonnen werden.

teilweise eine moderne Erklärung für konstitutionstherapeutische Ansätze über die Behandlung von Leber und Magen-Darm-Trakt an (gezielte Einflüsse über das enterale Nervensysten auf ZNS und vegetatives Nervensystem). Das betrifft z.B. die Versuche, auf solchen Wegen gezielt allgemeine tonisierende Wirkungen auszuüben („Kräf- tigung“ von Körper und Seele, positive Beeinflussung des Gesamtbefindens eines Menschen) [12,18,19,33]. In einem solchen Kontext können „Leberstörungen“, vor allem aber auch Funktionsund Motilitätsstörungen des Magens, als eine Art Dispositionsfaktor für seelische Beeiträchtigungen und Erkrankungen angesehen werden. So kann
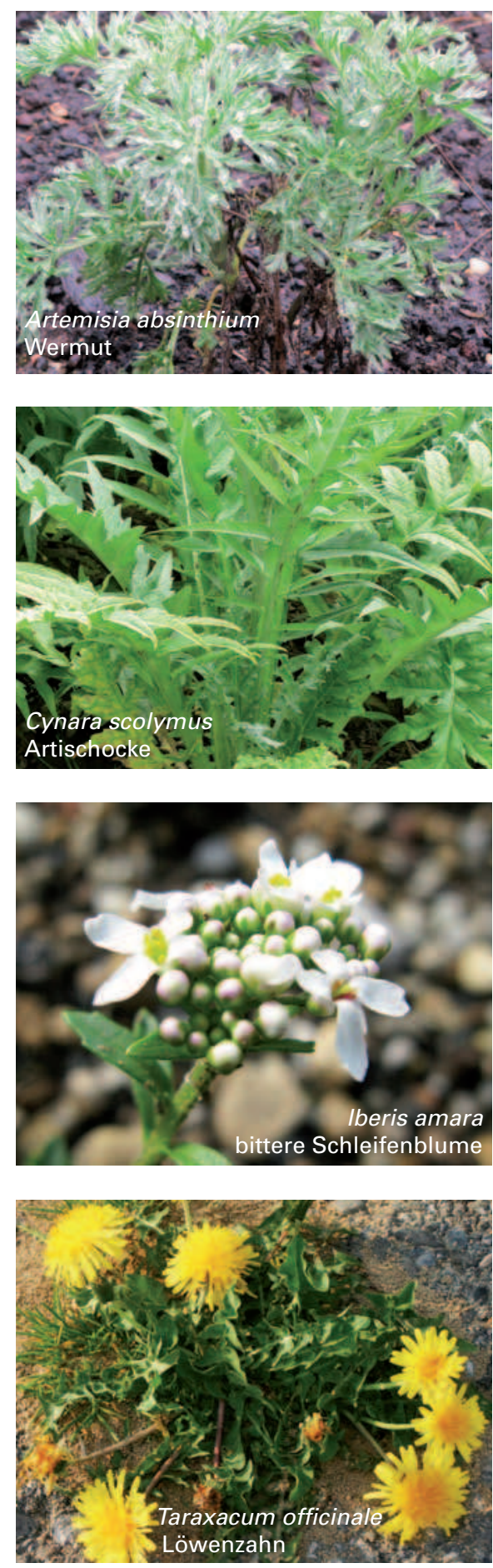

ein „atonischer Magen“ (gastrische Ursachen) Anlass für die Auslösung bzw. Verschlimmerung zahlreicher Erkrankungen und Beschwerden sein - nicht nur des Magen-Darm-Kanals und seiner Anhänge. Bittere phytotherapeutische Zubereitungen (z.B. Tinkturen) lassen sich dementsprechend bei Vorliegen von Funktionsstörungen von 
Magen, Darm, Leber und Galle für damit in Verbindung stehende extraintestinale Beschwerden therapeutisch einsetzen, z.B. bei bei Depression, Verstimmungen, Müdigkeit, Erschöpfung $[1,2,3,18,19,26]$. „Empirisch“ betrachtet hängen Verdauungs- und Funktionsstörungen des Magens häufig mit Obstipation zusammen, eher selten mit Diarrhöe.

Mit solchen Überlegungen können Erfahrungen erklärt werden, die darauf hinweisen, dass z.B. seelische Erkrankungen mitunter durch Störungen des Magen-Darmtraktes, aber auch Störungen von Leber und Galle, einen intensiveren und hartnäckigeren Charakter bekommen können und dass Behandlungsansätze über Leber und Magen-Darm-Trakt sinnvoll sein können.

Der Einsatz phytotherapeutischer Bitterstoffe lässt sich auch als eine Art „energetisierende“ Behandlung charakterisieren (siehe Tab. 4). In der Tabelle 5 sind ausgewählte Tinkturen entsprechend ihrer „Bitterkeit“ und damit assoziierend auch ihrer „Energetisierung“ gereiht (Wermutkrauttinktur als bitterste Tinktur und Schafgarbetinktur als am wenigsten bittere Tinktur).

$\mathrm{Zu}$ den Anwendungsmöglichkeiten könnten auch verschiedene somato- forme Störungen gehören (funktionelle Störungen), bei denen Störungen der körperlichen Funktionen bestehen, ohne dass ein „krankhafter“ organpathologischer Befund erhoben werden kann bzw. ohne dass eine organmedizinisch zentrierte Erklärung gefunden wird. Dabei finden sich häufig multiple und wechselnde körperliche Beschwerden („funktionelle Plastizität“) und ganz unterschiedliche Muster körperlicher Beschwerden. Ursachen, Entstehung und Aufrechterhaltung werden multifaktoriell vermittelt. Phytotherapeutische Vielstoffgemische mit einem Spektrum verschiedener Wirkmechanismen (Pleiotropie und MultiTarget-Charakter) könnten in der Behandlung einen wichtigen Platz einnehmen.

\section{Bitterstoffdrogen als eine Art von Adaptogenen}

Adaptogene machen den Organismus gegenüber physikalischem, chemischem, biologischem und psychosozialem Stress widerstandsfähiger (anpassungsfähiger?), d.h. sie erhöhen Adaptation und Toleranz gegenüber Stressoren. Adaptogene sollen eher „unspezifische“ Wirkungen zeigen, d.h. unab- hängig von der Art des pathologischen Zustandes „normalisierend“ (d.h. je nach Situation stimulierend oder beruhigend) wirken und die Körperfunktionen so wenig als möglich stören. Sie können bei Gesunden („Gesundheitsstärkung“, individuelle Primärprävention) wie bei Kranken (Therapie, individuelle Sekundärprävention) eingesetzt werden. Es stehen „Antistresswirkungen“ gegenüber nichtinfektiösen Stressoren im Vordergrund, einige Adaptogene zeigen auch gewisse immunmodulierende, nootrope oder anabole Effekte. Adaptogene Vorgehensweisen sind in vielen Medizinkulturen bekannt, z.B. Konzept von Asthenie Sthenie oder Hyper-, Normo-, Hypotonus (TEN, Traditionelle Europäische Naturheilkunde) oder ayurvedisch Rasayana.

\section{Bitterstoffdrogen: gastrointestinale Motilität und Sekretion}

Krankheits- vor allem aber therapiebedingt (z.B. durch bestimmte Zytostatika, anticholinerg wirksame Arzneimittel) kann die Funktion des gesamten Magen-Darm-Traktes erheblich beeinträchtigt sein (z.B. drastisch her-

Tab. 6. Magentee (I - VI) (Standardzulassung 1993)

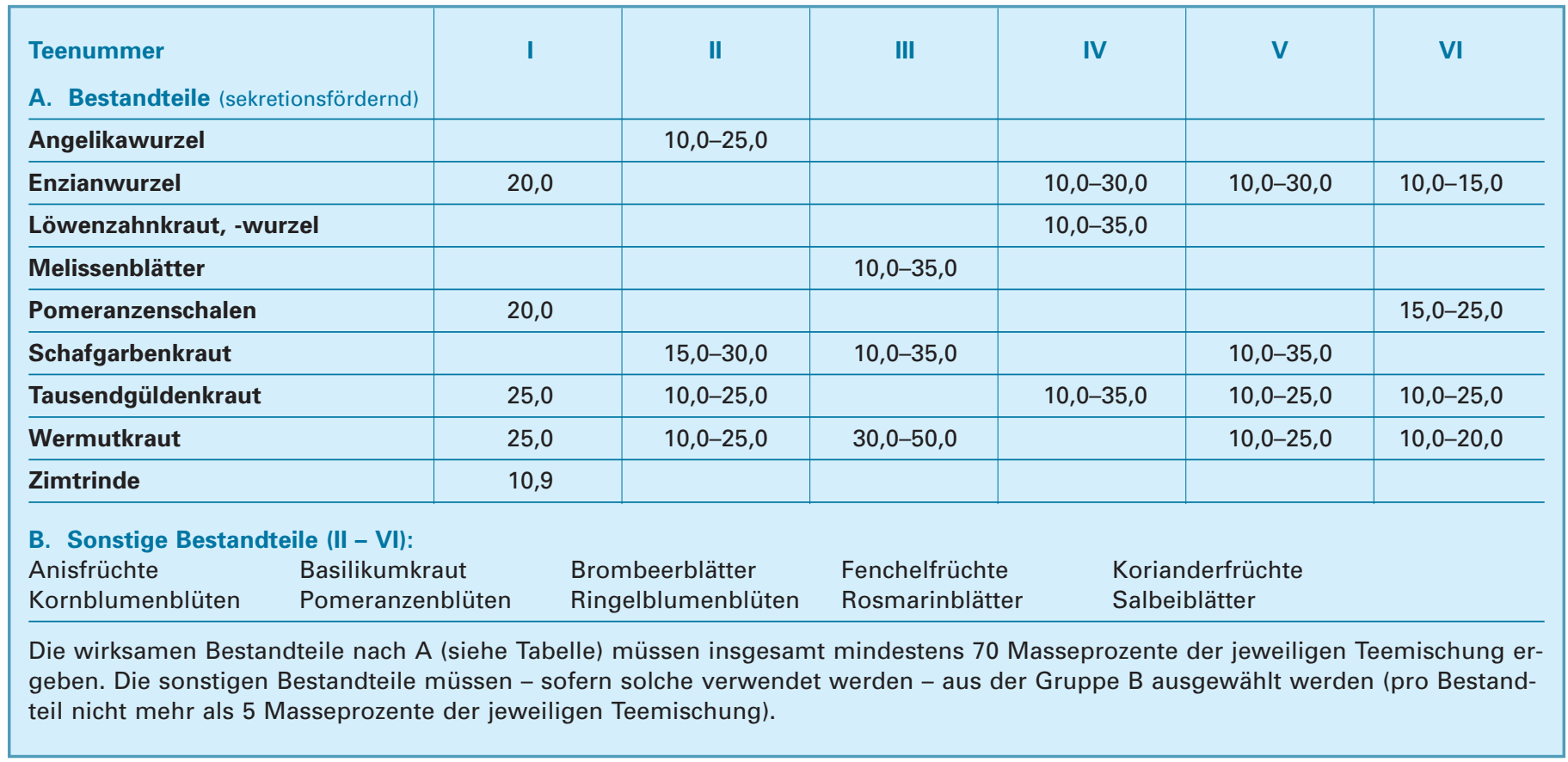


abgesetzte Motilität bzw. Hypermotilität oder erheblich verminderte bzw. übersteigerte Sekretion). Bitterstoffzubereitungen bzw. Kombinationspräparate mit Bitter- und Scharfstoffdrogen können eine herabgesetzte Motorik des Magen-Darm-Traktes (bis hin zur Gastrostase) günstig beeinflussen, ebenso auch die gastrointestinale Sekretion und damit auch Resorption und Verdauung. Hierzu liegen einige experimentelle Untersuchungen vor $[4,30,31$, 32]. Entsprechende Behandlungsversuche können daher bei einer verminderten Motorik, mitunter auch bei einer unkoordinierten Motorik, sinnvoll sein.

\section{Dosierung von Bitterstoffdrogen}

Sinnvolle Anhaltspunkte finden sich in verschiedenen zusammenfassenden Aufarbeitungen, z.B. für Extrakte aus Artischockenblättern bei 900-1800 mg (entsprechend 5-10 g Droge/Tag) [7, $11,20,29]$ oder mittlere Tagesdosen für Wermutkraut (z.B.verkapseltes Pulver) von 2-3 g Droge/Tag [7, 20,29,33]. Auch durch Teemischungen mit Bitterstoffdrogen lassen sich sowohl symptomorientierte wie auch konstitutionsorientierte Behandlungen durchführen (z.B. Magentees, siehe Tab. 6) $[1,2,3]$. Bei unmittelbar bitter schmeckenden Zubereitungen (Tees, Tinkturen) können v.a. für sensitive Patienten erheblich niedrigere Dosierungen angebracht sein, um Widerwillen und ggfs. Brechreiz zu vermeiden.

Je patientenzentrierter eine Behandlung geplant wird (konstitutionstherapeutische Gesichtspunkte) desto individueller erfolgen die Dosierung und Dosierungsanpassungen. Nicht selten scheinen z.B. bei Urtinkturen nur relativ niedrige Dosierungen notwendig zu sein, z.T. im Bereich von wenigen Tropfen/Tag [12]. Mittlerweile finden sich eine Reihe empirisch begründeter Tinkturen und Urtinkturen $[7,8,20,23$, $30,31,32,34]$, vor allem als Zubereitungen mit klarer wirkungsrelevanter Sensorik, z.B. verschiedene Bittermittel (Amara).

\section{Literatur}

1. Aschner B: Heilerfolge der Konstitutionstherapie bei weiblichen Geisteskrankheiten insbesondere bei Schizophrenie. Hippokrates Verlag, Stuttgart Leipzig 1933.

2. Aschner B: Lehrbuch der Konstitutionstherapie. 6. Aufl. Hippokrates Verlag, Stuttgart Leipzig 1934.

3. Aschner B: Technik der Konstitutionstherapie (mit 150 Fallbeispielen aus der Praxis). 7. Auflage, vollständig neu bearbeitet und ergänzt von Müller IW. Haug Verlag, Heidelberg 1995.

4. Blaschek W, Ebel S, Hilgenfeldt U, Holzgrabe $U$, Reichling J, Schulz V (Hrsg): Hagers Enzyklopädie der Arzneistoffe und Drogen. 2008. Hager ROM 2008; Springer electronic media. (http://www. Hagerrom.de).

5. Braun L, Cohen M: Herbs and natural supplements. An evidence-based guide. Churchill Livingstone, Sydney Edinburgh London New York 2005.

6. Drewnowski A (2001)The Science and Complexity of Bitter Taste. Nutr Rev 59:163169

7. European Scientific Cooperative on Phytotherapy (ESCOP): Monographs. The scientific Foundation for Herbal Medicinal Products. 2nd ed., completely revised and expanded. ESCOP - Thieme Verlag, 2003.

8. Gaby AR, Wright JV, Batz F, Chester R, Constantine G, Thompson LD (eds): The natural pharmacy. Revised and updated 3. edition. Three river press, New York 2006.

9. Iten F, Meier B, Saller R: Wirksamkeit von Teemischungen bei dyspeptischen Beschwerden - Eine Anwendungsbeobachtung. Forsch Komplementärmed Klass Naturheilkd 2002;9(5):277-282.

10. Jeon HJ, Kang HJ, Jung HJ, Kang YS, Lim CJ, Kim YM, Park EH (2008) Anti-inflammatory activity of Taraxacum officinale. J Ethnopharmacol 115:82-88.

11. Joy JF, Haber SL: Clinical uses of artichoke leaf extract. Am J Health Syst Pharm. 2007; 64:1904-1909.

12. Kalbermatten R: Wesen und Signatur der Heilpflanzen. Die Gestalt als Schlüssel der Heilkraft der Pflanzen. AT Verlag, Aarau 2002.

13. Mathew A, Taranalli AD and Torgal SS: Evaluation of anti-inflammatory and wound healing activity of Gentiana lutea rhizome extracts in animals. Pharm Biol 2004;42: 8-12.

14. Omer B, Krebs S, Omer H, Noor TO: Steroidsparing effect of wormwood (Artemisia absinthium) in Crohn's disease: A doubleblind placebo-controlled study. Phytomedicine 2007;14:87-95.

15. Oztürk N, Bafler KH, Aydin S, Oztürk Y, Califl I: Effects of Gentiana luteassp. symphyandra on the central nervous system in mice. Phytother Res. 2002;16:627-631.

16. Pieroni A Torry B: Does the taste matter? Taste and medicinal perceptions associated with five selected herbal drugs among three ethnic groups in West Yorkshire, Northern England. J Ethnobiol Ethnomed 2007:3:21.

17. Rozengurt E: Taste receptors in the gastrointestinal tract. I. Bitter taste receptors and alpha-gustducin in the mammalian gut. Am J Physiol Gastrointest Liver Physiol 2006;291 (2):G171-G177

18. Saller R: Die Leber aus komplementärmedizinischer Sicht. Ars medici Thema Phytotherapie 2009;1:6-12.

19. Saller R, Iten F, Reichling J: Dyspeptische Beschwerden und Phytotherapie - eine Übersicht über traditionelle und moderne Phy- totherapeutika. Forsch Komplementärmed Klass Naturheilkd 2001;8(5):263-273.

20. Saller R, Reichling J, Hellenbrecht D: Phytotherapie. Klinische, pharmakologische und pharmazeutische Grundlagen. Haug Verlag, Heidelberg 1995.

21. Saller R, Reichling J, Rostock M, Iten F, Melzer J: Gastrointestinale Symptome bei tumorkranken Menschen - phytotherapeutische Behandlungsmöglichkeiten. Schweiz. Zschr. GanzheitsMedizin 2008;20:221-230.

22. Schütz K, Carle R, Schieber A: Taraxacum - A review on its phytochemical and pharmacological profile. J Ethnopharmacol 2006;107: 313-323

23. Schulz H: Vorlesungen über die Wirkung und Anwendung der deutschen Arzneipflanzen. 2. Auflage. Georg Thieme Verlag, Leipzig 1929.

24. Sternini C: Taste receptors in the gastrointestinal tract. IV. Functional implications of bitter taste receptors in gastrointestinal chemosensing Am J Physiol Gastrointest Liver Physiol 2007;292(2):G457-G461.

25. Sweeney B, Vora M, Catherine Ulbricht C, Basch B: Evidence-Based Systematic Review of Dandelion (Taranacum officinale) by Natural Standard Research Collaboration. J Herb Pharmacother 2004;5:79-93.

26. Tharakan B, Manyam BV: Botanical therapies in chronic fatigue. Phytother. Res. 2006;20(2): 91-95.

27. Walker JM: The bitter remedy. Eur J Herbal Med 2003;6(2):28-33.

28. Weiss RF: Lehrbuch der Phytotherapie. 7 Aufl., Hippokrates, Stuttgart 1991.

29. Wichtl M (Hrsg): Teedrogen u. Phytopharmaka. 4. Aufl. Wissenschaftliche Verlagsgesellschaft, Stuttgart 2002.

30. World Health Organization: WHO monographs on selected medicinal plants. Volume 1. Geneva 1999.

31. World Health Organization: WHO monographs on selected medicinal plants. Volume 2. Geneva 2002

32. World Health Organization: WHO monographs on selected medicinal plants. Volume 3 Geneva 2007.

33. Zimmermann W: Praktische Phytotherapie. Die Arzneipflanzen der Medizin. Sonntag Verlag, Stuttgart 1994

34. Zizmann PA: Pflanzliche Tinkturen und Extrakte erfolgreich rezeptieren. Individuelle $\mathrm{Na}$ turheilmittel als zeitgemässe Therapie. Sonntag Verlag, Stuttgart 1991.

\section{Disclosure Statement}

The authors declare that no financial or other conflicts of interest exist in relation to the content of this article.

\section{Korrespondenzadresse}

Prof. Dr. med. Reinhard Saller Institut für Naturheilkunde Departement für Innere Medizin UniversitätsSpital Rämistrasse 100, CH-8091 Zürich reinhard.saller@usz.ch www.naturheilkunde.unispital.ch 